\title{
Sympathetic Regulation of Slc2a4 Gene Expression: Participation of a Putative cAMP Responsive Element (CRE) Site in the Slc2a4 Promoter
}

\author{
Ana Barbara Alves-Wagner Caio Yogi Yonamine Luciana Alves de Fatima \\ William Festuccia Ubiratan Fabres Machado \\ Department of Physiology and Biophysics, Institute of Biomedical Sciences, University of Sao Paulo, \\ Sao Paulo, Brazil
}

\section{Key Words}

Slc2a4 • CRE • CREB • Sympathetic activity

\begin{abstract}
Background/Aims: Studies have indicated that sympathetic activity enhances GLUT4 expression (Slc2a4 gene) by activating beta-adrenergic receptors. This could be mediated by a direct enhancer effect of cyclic AMP-responsive element binding protein (CREB) and family members upon Slc2a4 gene. However, a cAMP responsive element (CRE) in Slc2a4 promoter has never been demonstrated. Methods: Slc2a4 CRE-site was searched by in silico analysis. In skeletal muscles from rats displaying high sympathetic activity (SHR), Slc2a4 CRE-site was investigated by electrophoretic mobility shift assay (EMSA) and chromatin immunoprecipitation (ChIP) assay; and Slc2a4 expression was analyzed by RT-qPCR. Functional activity of the CRE-site was investigated by luciferase assay, 2 hours after 8-br-cAMP stimulation, in 3T3L1 adipocytes transientely transfected with native and mutated CRE-sites. Results: In silico analysis indicated the $-480 /-473$ segment as a putative CRE-site, with $62.5 \%$ of identity to CRE consensus sequence, and highly preserved in mouse, rat and human. CREB/CREM binding in this CRE-site was confirmed to occur in vitro (EMSA) and in vivo (ChIP assay). Enhancer activity of this segment in Slc2a4 transcription was confirmed in 3T3-L1 cells. Finally, in extensor digitorum longus muscle from SHR, 80\% increase in Slc2a4 mRNA expression was observed to be accompanied by increased CREB/CREM binding into the CRE-site both in vitro and in vivo. Conclusion: This study demonstrates the presence of a functional CRE-site at -480/-473 sequence of the Slc2a4 gene. This CRE-site has an enhancing activity on Slc2a4 expression and participates in the Slc2a4 increased expression observed in glycolytic muscles of rats displaying high sympathetic activity.
\end{abstract}




\section{Cellular Physiology Cell Physiol Biochem 2019;52:580-594 \\ \begin{tabular}{ll|l} 
and Biochemistry & $\begin{array}{l}\text { DOI: 10.33594/000000041 } \\
\text { Published online: } 22 \text { March } 2019\end{array}$ & $\begin{array}{l}\text { C } 2019 \text { The Author(s). Published by } \\
\text { Cell Physiol Biochem Press GmbH\&Co. KG }\end{array}$
\end{tabular} \\ Alves-Wagner et al.: CRE Site in the ScI2a4 Promoter Gene}

\section{Introduction}

Increased Slc2a 4 transcription, causing the solute carrier family 2 facilitated glucose transporter member 4 (GLUT4) protein content to increase in skeletal muscle and adipose tissue, is associated with an improvement in insulin-mediated cellular glucose disposal $[1,2]$. The opposite, i.e., a reduction in GLUT4 protein content is associated with insulin resistance and glucose intolerance $[1,2]$. Therefore, due to the pivotal role of GLUT4 in determining glycemic homeostasis, it is of great importance to understand the molecular mechanisms regulating Slc2a 4 transcription.

The Slc2a4 gene promoter contains several responsive elements to transcription factors, which can either activate or repress its transcription [3]. The transcriptional factors myocyte enhancer factor 2 (MEF2), myoblast determination protein 1 (MyoD) and thyroid hormone receptor alpha1 (TR $\alpha 1$ ) were shown to bind to -502 to -420 promoter region, and interact to synergistically enhance Slc2a4 transcription [4, 5]. Recently, our group has found that proinflammatory transcriptional factors nuclear factor NF-kappa-B (NFKB) and RELA proto-oncogene NF-KB subunit (RELA), p50 and p65 proteins; respectively, whose activation promotes insulin resistance, inhibit Slc2a4 transcription by directly binding to $-134 /-113$ and $-83 /-62$ regions of the Slc2a4 promoter [6].

Previous studies conducted by our group have gathered strong evidence indicating that the sympathetic nervous system plays a role in the regulation of GLUT4 content in skeletal muscle, an effect that seems, at least in part, to be due to the induction of Slc2a 4 expression [7, 8]. More specifically, we have found that the sympathetic nervous system, through activation of $\beta$-adrenergic receptors, maintains GLUT4 content in glycolytic skeletal muscle during fasting [7]. Indeed, pharmacological $\beta$-adrenergic receptor blockade with propranolol significantly reduced skeletal muscle Slc2a4 expression in fasted rats [7]. Further spontaneously hypertensive rats (SHR), which display peripheral sympathetic overactivity [9], showed increased Slc2a4 mRNA levels in both soleus and EDL skeletal muscles during experimental diabetes [8]. $\beta$-adrenergic signaling can modulate the transcription of genes that contain a cAMP responsive element (CRE) binding site in their promoter region. Generally, this involves catecholamines binding to $\beta$-adrenergic receptor, adenylate cyclase activation, elevation in intracellular cAMP content, protein kinase A (PKA) activation and phosphorylation of transcriptional factors CREB (cyclic AMP-responsive element binding protein) and family members CREM (CRE modulator) and ATF1 (activating transcription factor 1) [10]. Upon phosphorylation, those transcriptional factors, which remain bound to the CRE sequence even in the inactive state, change their conformation and interact with cofactors such as CBP and p300, thus promoting gene transcription [11]. Full consensus CRE consists of a palindromic nucleotide sequence TGACGTCA, but partial CRE sequences (TGACG or CGTCA) and some variants have also been shown to be functionally targeted by CREB [10]. Importantly, CREB, CREM and ATF1, which can bind as homo- or heterodimers in the CRE sequence of target genes [12], have a distinct pattern of expression throughout the body. While CREB and ATF1 are ubiquitously expressed, CREM expression is more restricted to neuroendocrine tissues [13].

CREB transcriptional activity has been pointed out as an important regulator of metabolic processes in different tissues. In skeletal muscle, for example, expression of a dominant negative polypeptide A-CREB was shown to cause dystrophy and extensive fiber necrosis [14]. In hepatocytes, CREB was shown to stimulate a gluconeogenic program by binding to the promoter of phosphoenolpyruvate carboxykinase and glucose-6-phosphatase genes during fasting, whereas, in pancreatic beta cells and adipocytes, it stimulates the IRS2 expression [10].

Considering the above-mentioned implication of $\alpha$-adrenergic activity in the regulation of glucose homeostasis and GLUT4 content in skeletal muscle, we tested the hypothesis that CREB is a positive regulator of Slc2a 4 transcription by binding to a CRE sequence located in the Slc2a 4 promoter region. 


\section{Cellular Physiology Cell Physiol Biochem 2019;52:580-594

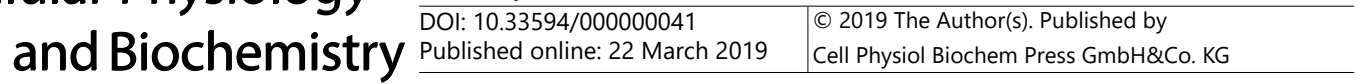 \\ Alves-Wagner et al.: CRE Site in the Scl2a4 Promoter Gene}

\section{Materials and Methods}

\section{Animals}

Animal handling and procedures were approved by the Ethical Committee for Animal Research of the Institute of Biomedical Sciences, University of Sao Paulo (Protocol \#059/2014). Male Wistar (W) and spontaneously hypertensive rats (SHR) from the Animal Facility of the Institute of Biomedical Sciences, University of Sao Paulo (Sao Paulo, Brazil), were kept on a chow diet (Nuvilab CR1, Nuvital Nutrients S/A, Parana, Brazil) and water ad libitum. Eight-week-old animals were killed by an overdose of sodium thiopental (60 mg/Kg, Cristália ${ }^{\circledR}$, Itapira, São Paulo, Brazil) for tissue harvesting. Soleus and extensor digitorum longus (EDL) skeletal muscles were collected and processed for further analysis.

\section{Cell culture}

Rat L6 cells were obtained from ATCC (Rio de Janeiro Cell Bank) and grown as previously indicated [6]. After $80 \%$ confluence, cells were maintained in differentiation media for 7 days and used for experiments. Mouse 3T3-L1 preadipocytes obtained from ATCC (Rio de Janeiro Cell Bank) were grown and differentiated to adipocytes as described before [6]. Eight-day differentiated 3T3-L1 cells, treated with $1 \mathrm{mM}$ of 8-br-cAMP (8-bromoadenosine 3', 5'cyclic monophosphate sodium salt, Sigma-Aldrich, St. Louis, MO, USA) for 2 h, or left untreated, were used for Slc2a4 expression analysis. Those cells were also used for luciferase assay, as described below.

\section{Real time-PCR analyses ( $q P C R$ )}

Soleus and EDL skeletal muscles and 3T3-L1 cells were subjected to total RNA extraction using Trizol Reagent ${ }^{\circledR}$ (Invitrogen, Carlsbad, CA, USA). Approximately $2 \mu \mathrm{g}$ of total RNA was reverse-transcribed using the kit ImProm II from Promega (Madison, WI, USA). The qPCR amplification was performed using Taqman ${ }^{\circledR}$ PCR master kit (Applied Biosystems Inc., Foster City, CA, USA) and carried out in the StepOne Plus Instrument (Applied Biosystems Inc.), as previously described [15]. Taqman ${ }^{\circledR}$ ID assays were AI5IQJM186914021_1, Rn00667869_m1, Mm01245502_m1 and Mm99999915_g1, respectively for rat Slc2a4, rat Actb, mouse Slc2a 4 and mouse Gapdh. The reference gene Actb was chosen for Slc2a4 analysis in rat skeletal muscle and L6 cells, and the reference gene Gapdh for mouse Slc2a4 analysis in 3T3L1 adipocytes, was based on the RefFinder software analysis. The method of $2^{-\Delta \Delta C t}$ was used for mRNA quantification.

\section{Electrophoretic Mobility Shift Assay (EMSA)}

Nuclear protein extraction and EMSA from skeletal muscles and L6 cells were carried out as previously described [6, 16]. Briefly, double-stranded oligonucleotide containing the putative CRE sequence of the Slc2a4 promoter gene was end-labeled with T4 polynucleotide kinase (Invitrogen, Carlsbad, CA, USA) and $\gamma^{-32} \mathrm{P}$ ATP (PerkinElmer Life, Waltham, MA, USA). Nuclear proteins were incubated with the labeled oligonucleotide probe in a buffer, for $20 \mathrm{~min}$ at room temperature. Competition binding experiments were performed under the same conditions, with the addition of 10 -fold molar excess of native and mutant (Mut1 and Mut2) unlabeled oligonucleotides. The oligonucleotides spanned the -489/-467 sequence of the Slc2a4 gene, and were described in the results. For antibody competition assay, nuclear proteins were incubated for $1.5 \mathrm{~h}$ with $10 \mu \mathrm{g}$ of anti-CREB/CREM antibody (ab5803, Abcam, Cambridge, MA, USA), before adding the probe. DNA/protein complexes were electrophoresed on $4 \%$ non-denaturing polyacrylamide gel at $4^{\circ} \mathrm{C}$. The gel was dried and exposed to a hyperfilm (Amersham Hyperfilm ECL, GE Healthcare Life Sciences) for 3 days at $-80^{\circ} \mathrm{C}$. The blots were analyzed by scanner densitometry (ImageScanner III, GE Healthcare, Sweden). Results were expressed as arbitrary units.

\section{Chromatin immunoprecipitation (ChIP) assay}

The ChIP assay was performed as previously described [6]. Briefly, skeletal muscle was pulverized in buffer (PBS 1X) and incubated in formaldehyde for $10 \mathrm{~min}$. The cross-link reaction was stopped with $0.125 \mathrm{M}$ glycine for 5 min. Samples were then mixed with lysis buffer (1\% SDS, EDTA 0.01 M, Tris-HCL 0.05 M, pH 8.1) for $2 \mathrm{~h}$, and subjected to DNA fragmentation by sonication, to obtain fragments of 200-1000 bp. One hundred microliters of the fragmented samples were diluted in buffer $(0.1 \%$ SDS, $1 \%$ Triton X-100, $0.1 \mathrm{M} \mathrm{NaCl}, 1 \mathrm{mM}$ EDTA, 20 mM Tris, pH 8.0) and pre-cleared with protein-A-sepharose (GE Healthcare, Buckinghamshire, UK) saturated with salmon sperm DNA for $4 \mathrm{~h}$. After that, an aliquot (10 $\mu \mathrm{L})$ was collected and used as 


\section{Cellular Physiology Cell Physiol Biochem 2019;52:580-594 \begin{tabular}{ll|l} 
DOl: 10.33594/000000041 & $\begin{array}{l}\text { O 2019 The Author(s). Published by } \\
\text { Cell Physiol Biochem Press GmbH\&Co. KG }\end{array}$
\end{tabular}

"input", and the supernatant of the sample was incubated with $10 \mu \mathrm{g}$ of anti-CREB/CREM antibody (ab5803, Abcam, Cambridge, MA, USA) or without antibody for $16 \mathrm{~h}$. Therefore, $65 \mu \mathrm{L}$ of protein-A-sepharose (GE Healthcare, Buckinghamshire, UK) saturated with salmon sperm DNA was added for $4 \mathrm{~h}$. The pellet was washed (low salt, high salt, LiCl, TE buffers) and added with elution buffer (1\% SDS, $0.1 \mathrm{M} \mathrm{NaHCO}$ ). The supernatant was treated with $\mathrm{NaCl} 5 \mathrm{M}$ (to crosslink reversal), RNase and proteinase K. DNA isolation, including the input aliquot, was performed by phenol:c hloroform:isoamylalcohol extraction and ethanol precipitation. Ten percent of total sample volume was subjected to DNA amplification, using Platinum $^{\text {TM }}$ SYBR $^{\mathrm{TM}}$ Green qPCR SuperMix-UDG (ThermoFisher Scientific, CA, USA), and primers sequences (forward, CCAGACACGGTTCTCAGACA; and reverse: GAAACAATGCCCCGAAGTAA) spanning the -670/-461 segment. Results were analyzed by $2^{\wedge \text {-(Ct Input - Ct IP) }} \mathrm{x} 100$, and the no antibody-treated sample value was subtracted to obtain the final data. To confirm the efficiency of immunoprecipitation, the PCR product from samples treated or not with anti-CREB/CREM antibody were electrophoresed on 1.5 ethidium bromideagarose gel, to visualize the amount of the CREB/CREM bound DNA.

\section{Luciferase assay}

A fragment of 962 nucleotides, spanning the -975/-14 region of the rat Slc2a4 gene promoter, was amplified by PCR with primers described in Table 1, and cloned in a directional manner using MluI and HindIII restriction sites upstream the luciferase gene in the pGL3-basic vector. Mutations in the Slc2a4 promoter CRE site located at position $-480 /-473$ were introduced using the primers described in Table 1 with the help of a mutagenesis kit (QuickChange XL Site-Directed Mutagenesis Kit, Agilent Technologies) following suppliers' protocol. The sequences of all constructs were confirmed by automated DNA sequencing. Plasmids were transformed into DH5 $\alpha$ bacteria, which were grown in LB containing ampicillin. Bacteria were harvested by centrifugation and plasmids were purified by maxi-prep (QIAfilter Plasmid Maxi Kit, QIAGEN) following suppliers' recommendations.

Differentiated 3T3-L1 adipocytes were transiently transfected (12-well plates) with $0.75 \mu \mathrm{g}$ of either an empty pGL3-basic (no promoter cloned in), or a wild type or a CRE-mutated Slc2a4 promoter pGL3basic, and $0.75 \mu \mathrm{g}$ of the internal control renilla expressing pRL-CMV vector, using Lipofect-AMINE 3, 000 (ThermoFisher Scientific), according to the manufacturer's protocol. The transfection was carried out overnight ( $\sim 16$ hours), and then, the cells were maintained in differentiation media for 48 more hours. After that, cells were treated or with 8-br-cAMP or left untreated, as described above in cell cultures, and evaluated for luciferase activity using the Dual-Luciferase ${ }^{\circ}$ Reporter Assay System (Promega, Madison, WI, USA), following the instructions of the manufacturer. The results were standardized for renilla luciferase activity.

\section{Data analysis}

All values were reported as mean \pm SEM. Two means were compared by unpaired two-tailed Student's t-test, and more than two means by one-way ANOVA, with Newman-Keuls posttest. Number of samples were supplied in the legends. The tests were performed using GraphPad Prism version 5.01 for Windows, GraphPad Software, San Diego, California, USA. 


\section{Results}

High beta-adrenergic activity enhances

Slc2a4 gene expression

Slc2a4 mRNA content was measured in skeletal muscle from SHR, a strain that features peripheral hyperactivity of the sympathetic nervous system [9, 17]. As depicted in Fig. 1A and 1B, SHR had higher Slc2a4 mRNA content in soleus $(\sim 35 \%)$ and EDL $(\sim 80 \%)$ skeletal muscles $(\mathrm{P}<0.001)$, as compared to Wistar rats. Additionally, in order to confirm the sensitivity of Slc2a4 gene expression to the intracellular $\alpha$-adrenergic signaling, 3T3-L1 adipocytes were treated with 8-br-cAMP for $2 \mathrm{~h}$. The Slc2a 4 mRNA increased by $30 \%(\mathrm{P}<0.01)$, reinforcing a role of $\alpha$-adrenergic signaling as an enhancer of Slc2a 4 gene expression.

\section{The 5'flanking region of the rat Slc2a 4 gene} contains one putative CRE site

By in silico analysis, we searched the presence of CRE consensus or homologous sequences [18], using the genome browser Ensembl Release 89 (http://www.ensembl.org/index.html). The $-480 /-473$ sequence of the rat Slc2a4 gene (positions according to the transcriptional start site) was identified as highly homologous to the previously described CRE-binding sites (Table 2 ). This segment shows $62.5 \%$ of identity to CRE consensus, and $87.5 \%$ compared to the CRE described in the phosphoenolpyruvate carboxykinase 1 (Pck1) gene and is highly conserved among rat, mouse and human genes (Fig. 2).

Since half CRE sequences (TGACG or CGTCA) were also proposed to be functional in some target genes, we also searched for these sequences in the 5'-flanking region of the mouse Slc2 a 4 gene. No half CRE sequences were found in the promoter region; however, a TGACG sequence is present downstream the ATG starting site, at position +30 to +34 of Slc2a 4 exon 1 .

\section{Skeletal muscle nuclear proteins bind}

selectively in the putative Slc2a 4 CRE

To demonstrate CREB/CREM binding in this region of the Slc2a4 promoter, we performed an EMSA using oligonucleotides (-489/-467) containing the putative wild type and mutated CRE sequences (Table 3). EMSA was performed with nuclear proteins from both rat skeletal muscle and L6 muscle cell (Fig. 3). We noticed the formation of 3 complexes of different sizes llanes

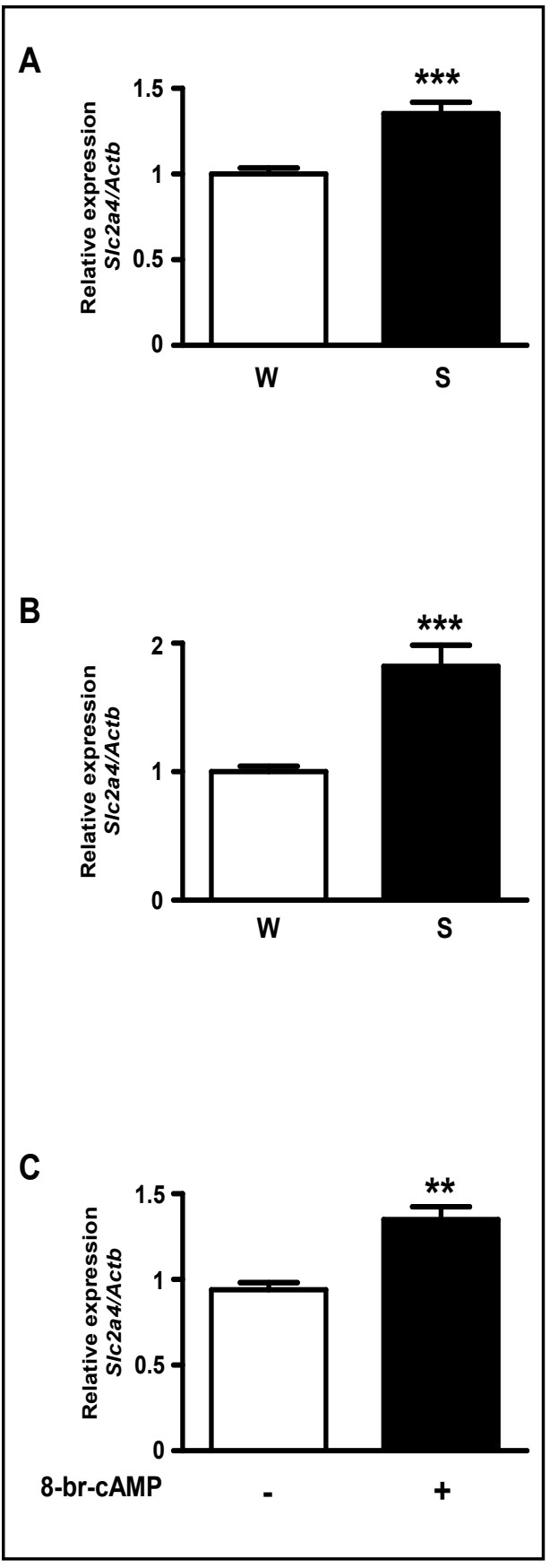

Fig. 1. High beta-adrenergic activity enhances Slc2a4 gene expression. Slc2a4 mRNA expression in soleus (A) and extensor digitorum longus (B) muscles of Wistar (W) and spontaneously hypertensive (S) rats, and in 3T3-L1 adipocytes after 2-h treatment with 8-br-cAMP (C). Data are mean \pm SEM of 8 to 9 animals in (A) and (B), and of 5 different samples in (C). ${ }^{* *} \mathrm{P}<0.01$ and ${ }^{* * *} \mathrm{P}<0.001$, unpaired two-tailed Student`s t-test. 
2, 5, 8 and 11). To show which complex depicts the specific binding into the putative CRE site, we performed a competition assay by adding an excess (10-fold molar) of unlabeled wildtype (lanes 3, 6, 9 and 12) or mutant oligonucleotides (lanes 4, 7, 10 and 13). The results show that addition of the unlabeled wild-type, but not unlabeled mutant oligonucleotides, markedly reduced the upper band (bigger DNA/protein complex) only, revealing that that band represents the putative CRE site of the Slc2a4 promoter.

Skeletal muscle CREB/CREM nuclear proteins selectively bind the Slc2a4 CRE in vitro and in vivo

To confirm the presence of CREB/CREM in the specific DNA/protein complex, in vitro, we performed the EMSA analysis with anti-CREB+CREM antibody (Fig. 4A). The presence of the antibody in the reaction almost abolished upper band formation in both skeletal muscle (lanes 2 and 4) and rat L6 cells (lanes 6 and 8). To demonstrate the binding activity of CREB/CREM in the Slc2a 4 promoter in vivo, we performed a ChIP assay in skeletal muscle extracted DNA. The fragmented DNA was immunoprecipitated with anti-CREB/CREM antibody, or left untreated, and a CRE-containing segment was amplified by qPCR. PCR from sample immunoprecipitated with anti-CREB/CREM revealed a clear band in ethidium bromide gel, as compared to sample treated without antibody (Fig. 4B). Quantification by qPCR revealed a $\sim 5$-fold increase $(\mathrm{P}<0.001)$ in the Slc2a4 segment amplification in samples immunoprecipitated with anti-CREB/CREM (Fig. 4C). Together, these results indicate the in vivo binding of CREB/CREM into the putative CRE sequence of the Slc2a4 promoter.

Slc2a4 CRE site participates on the CAMP-

stimulated transcriptional activity

To determine whether or not CREB/CREM binding into the CRE site affects the Slc2a4 transcription, we cloned -975/-14 segments of the Slc2a4 promoter, containing either a wildtype, or a partially deleted (6 nucleotides), or a completely deleted (8 nucleotides) or a mutated (4 nucleotides) CRE site (Table 4). These constructs were transiently transfected into 3T3-L1 cells, which were evaluated for luciferase activity after $2 \mathrm{~h}$ of stimulation with 8-br-cAMP. As depicted in Fig. 5, 3T3-L1 cells transfected wild-type CRE site displayed higher luciferase activity than those transfected with empty promoterless pGLBasic vector. Treatment with 8-br-cAMP significantly increased luciferase activity in cells transfected with the wild-type CRE $(\sim 70 \%)$, but not in cells transfected with the mutated CRE Slc2a4 sequences. Taken together, these results support a previously unrecognized direct activation of Slc2a4 transcription by transcription factors CREB/CREM under $\alpha$-adrenergic stimulation.

Table 2. In silico analysis identified the $-480 /-473$ sequence of rat Slc2a4 gene as highlyhomologous to the consensus CRE and its variants. The CRE consensus sequence was identified in somatostatin (Sst), parathyroid hormone (Pth), intracesternal A particle-promoted polypeptide (Ipp) genes; CRE variant sequences were described in Pck1, phosphoenolpyruvate carboxykinase 1; Fos, fos proto-oncogene; Penk, proenkephalin; Cxcl8, c-x-c motif chemokine ligand 8 (former interleukin 8); $G 6 p c$, glucose-6-phosphatase catalytic subunit genes [18, 22, 23]

\begin{tabular}{lcc}
\hline Genes & CRE sequence & \% of identity \\
\hline Sst, Pth, Ipp & TGACGTCA & 100 \\
Pck1 & TTACGTCA & 87.5 \\
Fos & TGACGTAG & 75.0 \\
Penk & CTGCGTCA & 62.5 \\
Cxcl8 & TGACATAA & 75.0 \\
G6pc (CRE1) & TTGCATCA & 62.5 \\
G6pc (CRE2) & TTACGTAA & 75.0 \\
Slc2a4 & TTACTTCG & 62.5 \\
\hline
\end{tabular}

Fig. 2. Putative cAMP responsive element (CRE) in the Slc2a4 gene. Conservation of the putative Slc2a4 CRE sequence (in bold) in the Slc2a4 gene of rat, mouse and human.

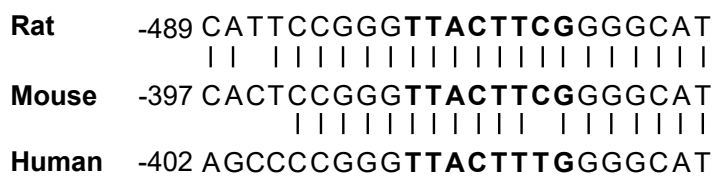




\section{Cellular Physiology \begin{tabular}{ll|l} 
and Biochemistry & Dublished online: 22 March 2019 & $\begin{array}{l}\text { C } 2019 \text { The Author(s). Published by } \\
\text { Cell Physiol Biochem Press GmbH\&Co. KG }\end{array}$
\end{tabular} \\ Alves-Wagner et al.: CRE Site in the Scl2a4 Promoter Gene}

In vitro nuclear protein binding activity in the Slc2a4-CRE site increases in extensor digitorum longus (EDL) of spontaneously hypertensive rats (SHR)

To confirm that a classical situation of high sympathetic activity, as in SHR, stimulates the nuclear protein binding activity into the Slc2a 4 CRE site, in vitro, an EMSA analysis was performed. As depicted in Fig. 6, binding activity was increased $(20 \%, \mathrm{P}=0.0191)$ in EDL (Fig. 6A), but not in soleus (Fig. 6B) muscles of SHR.

In vivo CREB/CREM binding activity increases in extensor digitorum longus (EDL) of spontaneously hypertensive rats (SHR)

To confirm that nuclear protein binding activity in the Slc2a4 CRE site also occurs in vivo, and involves CREB/CREM transcriptional factors, a ChIP assay was performed. In parallel to EMSA results, the ChIP assay revealed that the spontaneous in vivo CREB/CREM binding in the Slc2a4 CRE site was increased $(120 \%, \mathrm{P}=0.0456)$ in EDL (Fig. 7A), but not in soleus (Fig. 7B) muscle of SHR.

\section{Discussion}

In this study, we investigated the presence of a functional cAMP responsive element (CRE) in the Slc2a4 gene promoter, as well as whether $\alpha$-adrenergic activity regulates or not Slc2a4 gene transcription in a CREB/ CREM-mediated way. Our main findings indicate that the $-480 /$ 473 region of the Slc2a4 promoter is a CRE binding site, to which CREB/CREM proteins bind and enhance the Slc2a4 transcriptional activity. Furthermore, we confirmed that this CRE site is involved in the increase of Slc2a 4 mRNA expression, acutely induced by a cAMP analog in vitro, and chronically induced in glycolytic skeletal muscle of rats displaying high sympathetic activity.
Table 3. Description of wild type (WT), mutants 1 and 2 (Mut1 and Mut2) sequences of the $-489 / 467$ sequence of rat Slc2a4 promoter gene. The putative CRE is underlined, and the lowercase letters indicate the altered nucleotides in the Mut1 and Mut2 oligonucleotides

\begin{tabular}{lc}
\hline Oligonucleotide & Rat $-489 /-467$ Slc2a4 gene sequence \\
\hline WT & CATTCCGGGTTACTTCGGGGCAT \\
Mut1 & CATTCCGGGccACTcCGGGGCAT \\
Mut2 & CATTCCGGGTTcCTTtaGGGCAT
\end{tabular}

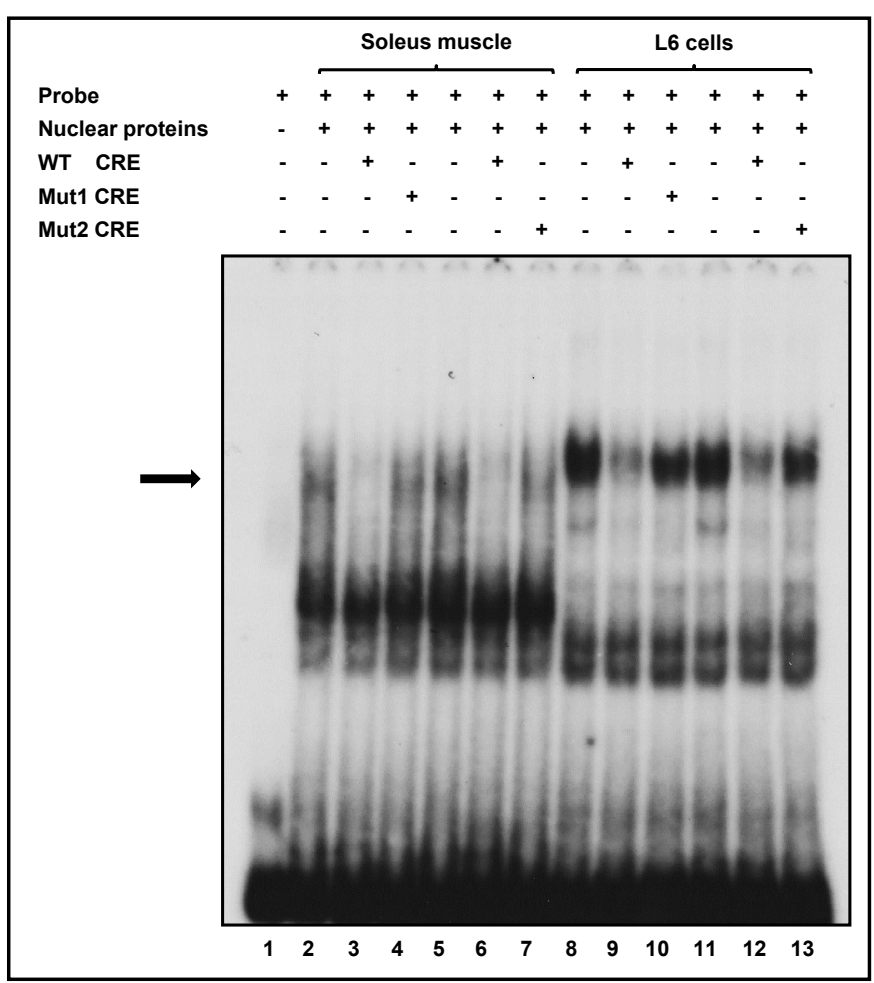

Fig. 3. Skeletal muscle nuclear proteins bind selectively in the putative Slc2a4 CRE. Nuclear proteins extracted from rat soleus muscle and L6 muscle cells were subjected to electrophoretic mobility shift assay to determine the binding activity into the putative CRE site of the rat Slc2a4 promoter. Nuclear protein extracts $(20 \mu \mathrm{g})$ were incubated with the radiolabeled wild type (WT) Slc2a4 CRE containing domain (probe); specific binding competitions were performed using 10-fold molar excess of unlabeled WT CRE, mutant 1 CRE (Mut1) and mutant 2 CRE (Mut 2). Representative image of the EMSA; the arrow indicates the specific protein/DNA band. 
Fig. 4. Skeletal muscle CREB/ CREM nuclear proteins bind selectively in the Slc2a4 CRE in vitro and in vivo. In vitro binding activity $(\mathrm{A})$ of nuclear proteins from rat soleus and L6 muscle cells was analyzed by electrophoretic mobility shift assay, using the wild type CRE binding site of the rat Slc2a 4 promoter as probe, and competitions were performed by adding $10 \mu \mathrm{g}$ of anti-CREB/ CREM antibody (CREB, CAMP responsive element binding protein; CREM, CRE modulator protein). In vivo CREB/CREM binding activity (B and $\mathrm{C}$ ) into the CRE binding site of the rat Slc2a 4 promoter gene was analyzed by chromatin immunoprecipitation (ChIP) assay. DNA was extracted from soleus muscle, fragmented, immunoprecipitated with anti-CREB/CREM antibody or not (No ab), and the -670/461 sequence of Slc2a4 gene was amplified by qPCR. To confirm the efficiency of immunoprecipitation, the PCR products were electrophoresed on ethidium

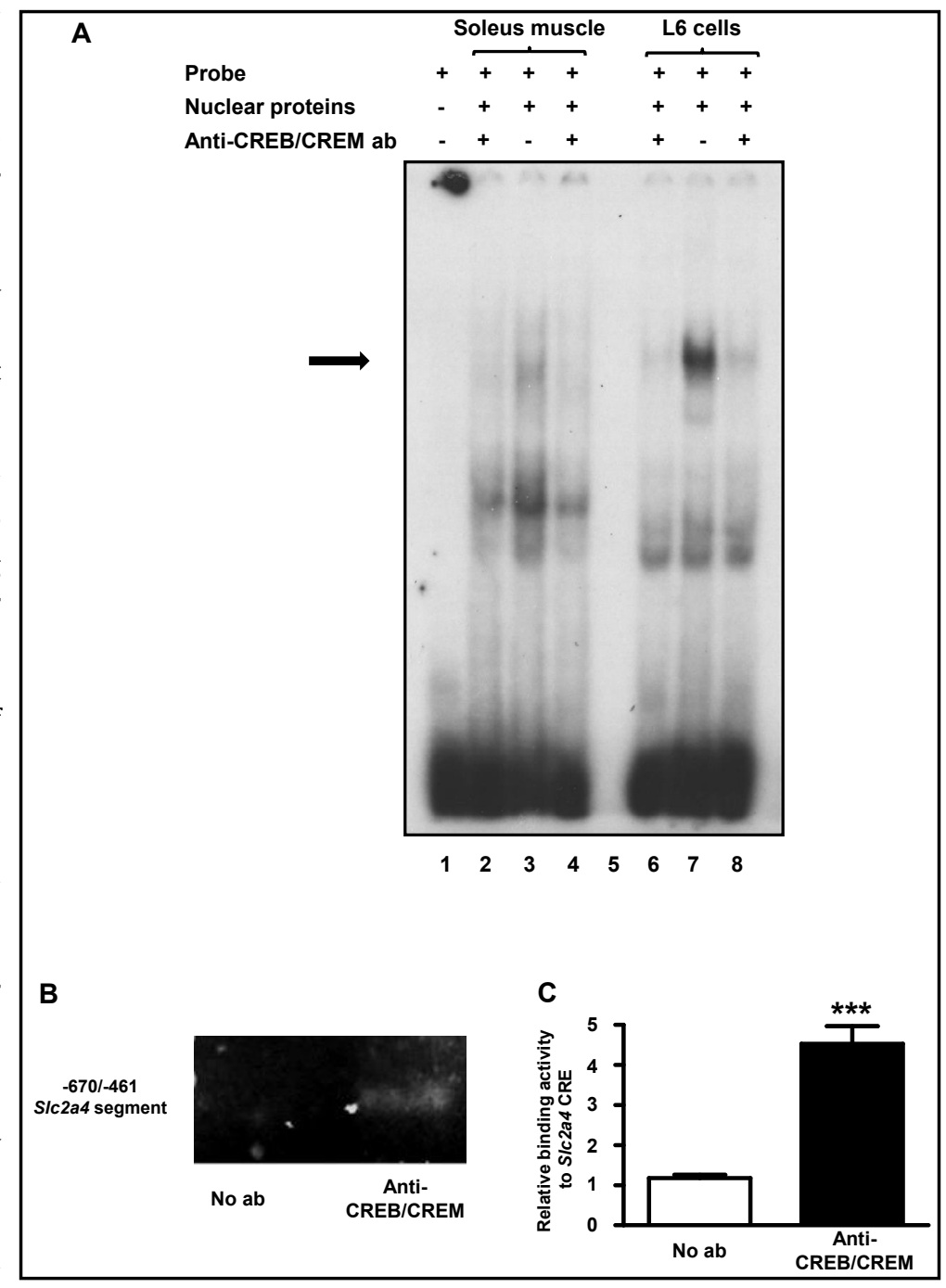
bromide-agarose gel (B) or quantified in the qPCR (C). A and B, representative images; C, mean \pm SEM of samples from 3 animals, treated or not with antibody. ${ }^{* * *} \mathrm{P}<0.001$, unpaired two-tailed Student`s t-test.

It is well-accepted that CREB and CREM are effective enhancers of most of their target genes [10], as we are now reporting for Slc2a4 gene. Montminy and colleagues, through in silico analysis, have identified 10, 447 full CRE consensus sequences (TGACGTCA) in the human genome [19]. However, despite the observation that unmethylated functional CREB-binding sites can be found in $\sim 5,000$ genes, exposure to cAMP stimulates the expression of only about 100 CREB target genes [10]. Nevertheless, some studies have already proposed a CREB/ATF3 repressor effect upon Slc2a4 gene [20, 21], as it will be discussed below.
Table 4. Description of wild type (WT) and mutant (Mut3, Mut4 and Mut5) CRE domains of Slc2a4 promoter transfected in 3T3-L1 adipocytes. Wild type (WT), 6 nucleotides deleted (Mut3), 8 nucleotides deleted (Mut4) and 4 nucleotides mutated (Mut5) CRE sequences (underlined) of the transfected Slc2a4 (dashes indicate deleted nucleotides and lowercase letters indicate mutated nucleotides)

\begin{tabular}{|c|c|}
\hline Primer name & $\begin{array}{l}\text { CRE domains of Slc2a4 promoter } \\
\text { transfected for luciferase assay }\end{array}$ \\
\hline WT & GGGTTACTTCGGGG \\
\hline Mut3 & GGGT _..... GGGG \\
\hline Mut4 & GGG $\ldots \ldots$ GGG \\
\hline Mut5 & 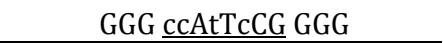 \\
\hline
\end{tabular}


Fig. 5. Slc2a4 CRE site participates in the cAMP-stimulate transcriptional activity. Luciferase activity of 3T3-L1 adipocytes transfected with Slc2a4 promoter sequence containing WT, Mut3, Mut4 and Mut5 CRE domains, and stimulated with 8-br-cAPM for $2 \mathrm{~h}$; basal transcriptional activity of cells transfected with the empty vector is shown on the left. Data are means \pm SEM of 3 different experiments. $\# \# \# \mathrm{P}<0.001$ vs all groups, one-way ANOVA, Student Newman Keuls post hoc test.

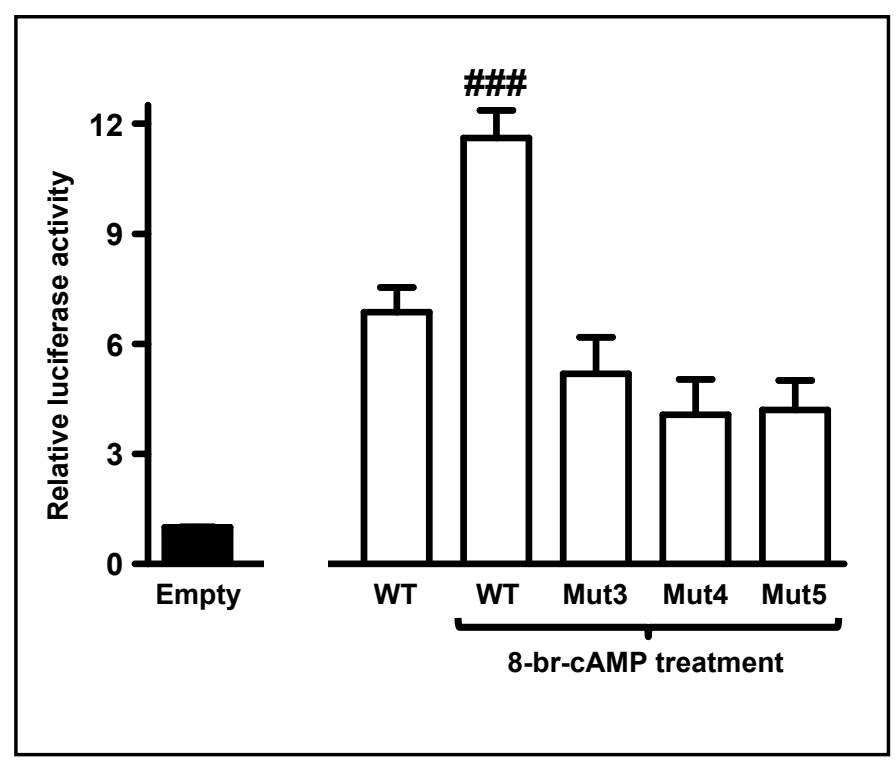

Fig. 6. In vitro nuclear protein binding activity into the Slc2a4 CRE increases in extensor digitorum longus (EDL) of spontaneously hypertensive rats. Nuclear proteins from extensor digitorum longus (A) and soleus (B) muscles of Wistar (W) and spontaneously hypertensive $(\mathrm{S})$ rats were subjected to electrophoretic mobility shift assay (EMSA), using a Slc2a4-CRE containing oligonucleotide as probe. In each panel, on the left, an EMSA image, on the right, quantification of the relative binding activity. Data are mean \pm SEM of 3 animals. ${ }^{*} \mathrm{P}<0.05$, unpaired two-tailed Student's t-test.

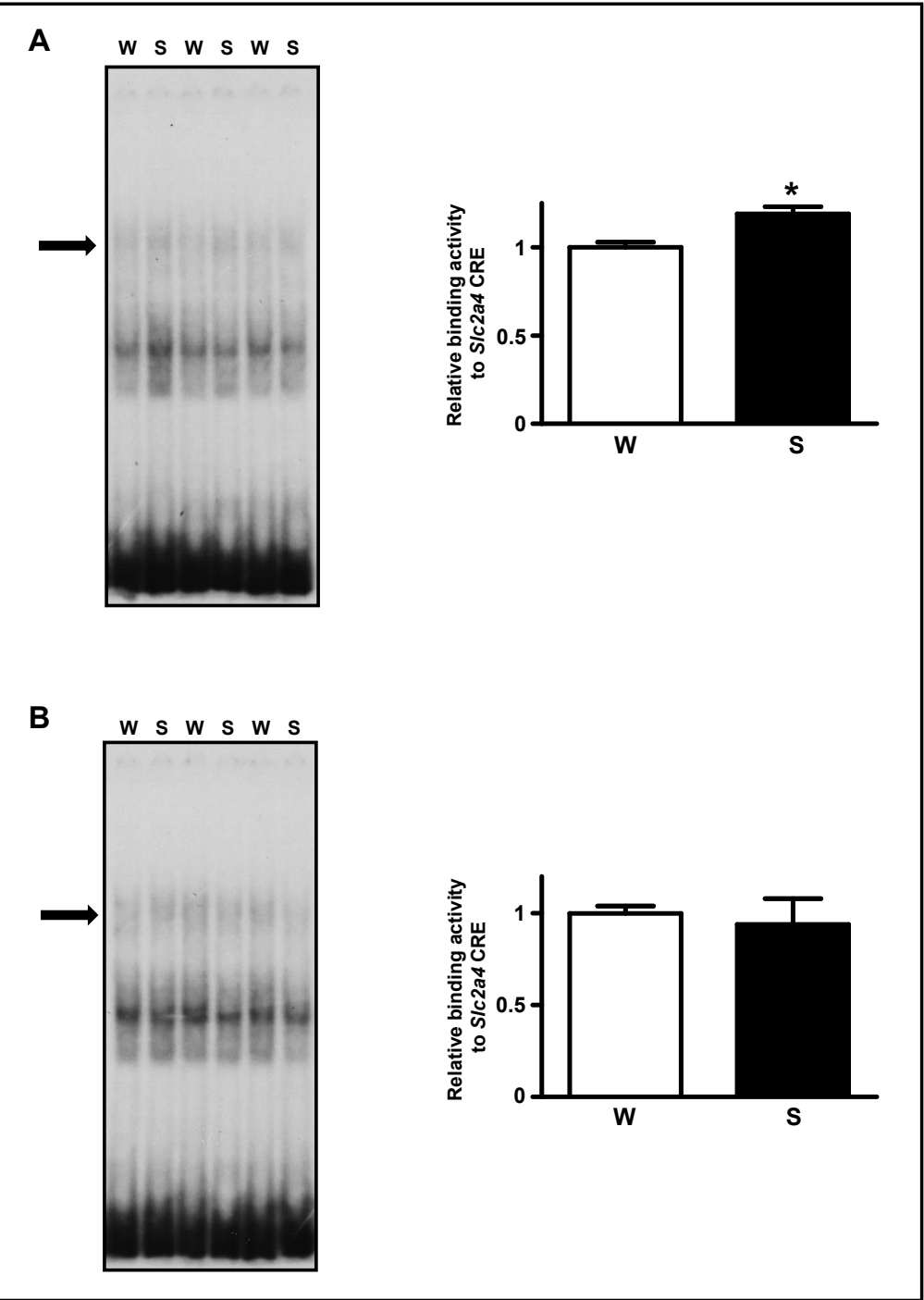


Fig. 7. In vivo CREB/CREM binding activity into the Slc2a4 CRE increases in extensor digitorum longus (EDL) of spontaneously hypertensive rats. CREB/ CREM binding activity into de Slc2a4-CRE binding site was evaluated by chromatin i m m unoprecipitation

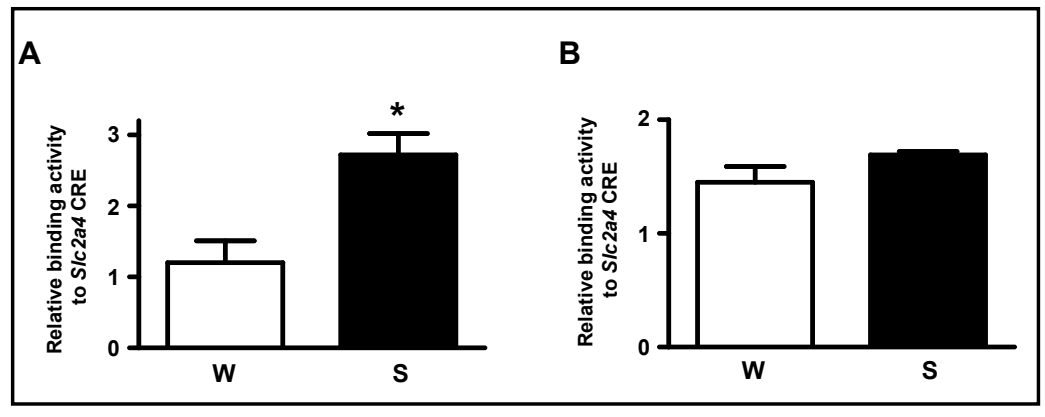
(ChIP) assay in EDL (A) and soleus (B) muscles from Wistar (W) and spontaneously hypertensive (S) rats. Muscle DNA was extracted, fragmented, immunoprecipitated with anti-CREB/CREM antibody or not, and the -670/-480 sequence of Slc2a4 gene was amplified by qPCR. Results of antibody-treated samples were subtracted for calculation of the final relative binding activity. Data are mean \pm SEM of 3 animals. ${ }^{*} \mathrm{P}<0.05$, unpaired two-tailed Student`s t-test.

Although Slc2a4 CRE-binding sites have already been proposed, their sequence, location and functional activity have never been extensively investigated. The CRE consensus sequence TGACGTCA is not present in the Slc2a 4 promoter. However, we identified and investigated a putative variant of CRE (TTACTTCG), located at the -480/-473 segment of the rat Slc2a4 gene promoter. This non-canonical CRE sequence displays $62.5 \%$ homology with the consensus CRE sequence, and is well-conserved among species such as rat, mouse and human.

Other CRE variant sequences have been reported as functional, most of them showing 62.5 to $87.5 \%$ homology to the consensus sequence $[18,22,23]$. Regarding the Slc2a4 gene, a putative CRE variant sequence (TTAAGTCA) was proposed, by in silico analysis, in the Slc2a4 gene of the teleost fish Fugu [24]; however, this sequence has never been investigated either as target for CREB/CREM proteins, as sensitive to cAMP, or as a regulator of Slc2a4 transcription. Furthermore, an allusion to the presence of an ATF3 binding site, between $-555 /-508$ region of the Slc2a4 gene, was made [20]; however, once more there was no demonstration that this domain binds the ATF3 or displays Slc2a4 transcriptional regulation. In fact, there is neither CRE consensus sequence nor highly homologous variant sequence in that segment referred to.

Half CRE sequences (TGACG or CGTCA) have also been proposed as potential sites for CREB/CREM regulation of target genes [10], but in silico analysis of rat Slc2a4 promoter did not reveal any half CRE. That highlights that every CREB/CREM-mediated regulation of Slc2a4 gene must be related to the CRE site we are reporting.

We started the characterization of that putative CRE-binding site by EMSA. We found that nuclear proteins from soleus muscle tissue or L6 muscle cells specifically bind an oligonucleotide containing the Slc2a4-CRE in vitro. Three DNA/protein complexes were observed, and by using oligonucleotides carrying mutations in the core of the putative CRE (TTAAGTCA), it was observed that the heavier complex must be related to CREB/ CREM binding. Indeed, the addition of anti CREB/CREM-antibody drastically decreased the intensity of this upper band, in both muscle tissue and cells, proving that this complex contains CREB/CREM proteins.

The classic result of antibody competition in EMSA is the supershift band, for which the antibody binds to the transcriptional factor, without interfering with the protein/DNA binding, thus increasing the molecular weight of the complex. However, it has become more evident that the addition of the antibody in the reaction can compete with the DNA, shifting the binding, and thus weakening the band. This type of result has already been observed for other transcriptional factors that regulate the Slc2a4 gene such as the myocyte enhancer factor 2D (MEF2D), hypoxia inducible factor 1A (HIF1A) and nuclear factors kappa B p50 and p65 [6, 25]. 
Subsequently, to confirm that CREB and CREM bind the Slc2a4 promoter in vivo, we performed a ChIP assay in soleus muscle samples. By immunoprecipitating CREB/ CREM cross-linked to the chromatin, we confirmed that these transcriptional factors bind endogenously to the Slc2a 4 CRE site. Increased forskolin-induced content of Slc2a 4 promoter bound to ATF3, analyzed by ChIP assay, was observed in HEK393T cells [20]. However, except for one ethidium bromide stained image, no additional information is provided to the reader concerning this result and its respective methodological details [20].

Finally, for functional characterization of the Slc2a 4 gene CRE site, luciferase activity was evaluated in the target cell 3T3L1 adipocyte, transfected with the -975/-14 Slc2a4 segment, mutated at the CRE site. Two-hour stimulation with 8-br-cAMP increased the luciferase activity of the wild type promoter; however, all CRE-mutated promoters did not respond to the cAMP analogue. Those results clearly show a rapid CRE-mediated enhancer effect on Slc2a4 gene transcription, pointing out that the effect observed was induced by the native cellular mediators of cAMP activity, and in a classical target cell such as the adipocyte.

Interestingly, the CRE site we characterized is located in the -500/-400 Slc2a4 promoter region which includes the binding sites of several other enchancers such as MEF2, MyoD, TR $\alpha 1$, LXR [5, 26, 27], and that makes probable cooperative interactions among these transcription factors. Particularly, the TRE and LXRE sites $[5,26]$ overlap the CRE site we are characterizing, and competitivity and/or cooperativity among these transcription factors deserves future studies.

Curiously, opposing regulation of CREB/ATF3 upon Slc2 a 4 gene transcription (inhibition) has already been proposed. However, this suggestion was based mainly on GLUT4 protein increase in adipose tissue of a high-fat-fed transgenic mice expressing a dominant negative CREB inhibitor protein (ACREB) in adipose tissue, or in ATF3\% mice [20]. In those transgenic animals, several intermediary modulators of Slc2a4 expression can be involved; and thus, these observations should be carefully analyzed, before the proposal of a direct CRE-mediated effect. Unexpectedly, the ACREB protein-induced increase in GLUT4 was shown only in high fat-fed mice [20], a condition that classically is known to repress GLUT4 expression [28, 29]. Additionally, this study [20] clearly demonstrated that in ACREB mice the high fat-induced inflammatory activity in adipose tissue was substantially reduced, a condition well-known to enhance Slc2a4 gene expression, since the inflammatory mediator nuclear factor kappa B is a powerful inhibitor of the Slc2a4 gene transcription [6]. Furthermore, transfected HEK293T cells, supposedly overexpressing A-CREB or ATF3 and a Slc2a4-luciferase reporter, subjected to 4-hour treatment with forskolin, were also used to propose the CRE-mediated repressor effect [20], but that could be an indirect effect.

Another attempt to demonstrate an ATF3/CRE-mediated repressor effect upon Slc2a4 expression was conducted in 3T3-L1 adipocytes, in which overexpression or silencing of the inducible cAMP early repressor (ICER) was accompanied by increased and decreased Slc2a 4 mRNA expression [21], respectively. However, in that study, no investigation concerning a direct ATF3/CRE-mediated repressor effect upon Slc2a4 transcription was conducted, and conclusions were based on the indirect ICER-mediated changes in intracellular cAMP content.

Considering the discussion above, reports of CREB/ATF3-CRE-mediated repressor effect upon Slc2a4 expression could be an indirect effect triggered in highly manipulated biological systems, instead of a direct CRE-mediated regulation of Slc2a4 gene, as we are reporting here in wild target cell.

Over the years, we have gathered evidence indicating that the sympathetic nervous system, by regulating Slc2a4 expression, plays an important role in controlling GLUT4 protein content in skeletal muscle. Indeed, we have shown that the sympathetic nervous system, through the activation of $\beta$-adrenergic receptors, stimulates Slc2a4 mRNA expression in glycolytic and oxidative skeletal muscles during fasting [7], and that the spontaneously hypertensive rat (SHR), which displays peripheral sympathetic overactivity [9], also shows increased expression of Slc2a4 in muscle [8]. In the present study, by RT-qPCR, we confirmed that SHR have increased Slc2a 4 expression in both oxidative and glycolytic muscles. Besides, 
the participation of the CAMP in this regulation was reinforced by the demonstration that 3T3-L1 cells treated with 8-br-cAMP for 2 hours increase the Slc2a 4 mRNA expression. These results demonstrate that high sympathetic activity increases Slc2a 4 expression, evincing this regulation as a cAMP-mediated effect.

Curiously, in contrast to our results, it has been previously reported that prolonged in vitro treatments ( 24 to $48 \mathrm{~h}$ ) of adipose or muscle cells with cAMP inhibit rather than enhancing the Slc2a4 expression [30,31]. However, experimental conditions of quantitative analysis of mRNA expression were quite inaccurate in this era, results were restricted to 3 samples only, without endogenous control, and none of those studies investigated whether this response was due to a direct action of CREB upon a CRE site in the Slc2a4 gene. Indeed, long-term treatment of 3T3-L1 adipocytes with 8-br-cAMP was reported to repress the Slc2a 4 gene expression by a mechanism involving the nuclear factor 1B (NF1B), which binds into the -706/-676 5'-flanking region of the murine Slc2a4 gene [32]; a region which does not match the CRE region we are reporting now. So, we propose that the repressor effect on Slc2a4 expression described earlier, and apparently related to cAMP, does not represent a direct CREB/CREM-mediated regulation in a CRE site; but a specific in vitro effect observed in response to long-term treatment with 8-br-cAMP, involving NF1B activation.

In order to demonstrate that high sympathetic activity enhances Slc2a4 expression in skeletal muscles of SHR by a CRE-mediated mechanism we measured the CREB/CREM binding activity in the Slc2 a 4 promoter. In EDL muscle of SHR, a higher CREB/CREM binding activity in the Slc2a4 CRE was observed, both in vitro (EMSA) and in vivo (ChIP), and this definitively reveals the participation of CRE activity in the Slc2a4 gene expression observed under this condition. However, increased Slc2a4 mRNA expression was also detected in soleus muscle of SHR, despite the fact that CRE binding activity was unaltered, suggesting that the CREB/CREM regulation of Slc2a4 gene in this muscle is not as important as in the EDL muscle.

Comparing soleus and EDL, distinct regulations of Slc2a4 expression have been described under conditions in which sympathetic activity is altered such as fasting [7], diabetic SHR [8] and insulin-treated diabetic Wistar rats [17]. Glycolytic muscles, such as the EDL, obtain energy primarily by glycolysis, using glucose as the main fuel, whereas oxidative muscles, such as soleus, have high capacity to also use fatty acids to generate ATP, reducing their need for glucose $[33,34]$. It is reasonable to understand that after prolonged fasting, GLUT4 expression is decreased in adipose tissue and red muscle, contributing to maintaining glycemia; but it is preserved in white muscle [35]. Maintenance of GLUT4 expression in glycolytic muscles during fasting was described as related to the beta-adrenergic activity [7]. This regulation highlights the relevance of GLUT4 to guarantee glucose-dependent glycolytic activity of fast-twitch fibers, which is fundamental to "fight-or-flight" responses controlled by the SNS, and likely played an important role in the evolution of humans. Regarding that, it is noticible that in evolutionary terms, the glycolytic fiber seems to appear after the oxidative fibers, as it does in the vertebrate's ontology, and this is a process related to motoneuron control [36].

In addition, although oxidative muscle displays a greater in vivo sensitivity to catecholamines [37], the effect of beta-adrenergic antagonists is higher in glycolytic fiber $[38,39]$, reinforcing that CREB/CREM-mediated regulation of Slc2a 4 may be most important in glycolytic fibers. However, although CREB/CREM binding into Slc2a 4 is unaltered in soleus muscle, we cannot discard CREB/CREM-induced indirect regulation of the Slc2a4 gene. For instance, CREB/CREM was reported to increase the transcriptional activity of the myocyte enhancer factor 2 (MEF2) [14], which is a powerful enhancer of Slc2a4 gene expression in soleus muscle $[5,16,25]$.

We might also analyze the possibility of some CRE-mediated alpha-adrenergic regulation of Slc2a4 expression, since alpha 2-AR is a Gi-protein-coupled receptor, and it decreases cAMP generation. However, it is known that beta1/2/3-adrenergic activities are predominant in muscle and adipose cells $[40,41]$, and some alpha-adrenergic effects may 
occur in muscle and adipose tissues just by altering blood flows, since alpha-ARs are highly expressed in the arteries [42].

\section{Conclusion}

This study demonstrates the previously unrecognized presence of a CRE site TTACTTCG at the $-480 /-473$ region of the Slc2a4 promoter gene. This CRE site is functional, displaying an enhancer role upon Slc2a4 gene transcription. This study demonstrates that the high sympathetic activity found in glycolytic muscle of SHR increases Slc2a4 transcription, and this regulation is associated with increased CREB/CREM binding into the Slc2a4 CRE site measured both under in vitro and in vivo conditions. Together, these results highlight the important role of cAMP-mediated increase in Slc2a4 expression in glycolytic muscles, which is fundamental to ensure metabolic support during a contractile response in stressful situations, such as hunting or escaping.

\section{Acknowledgements}

This research was supported by São Paulo Research Foundation (FAPESP) \#2012/04831-1. ABA-W was recipient of a FAPESP fellowship \#2013/26616-8. We thank Dr. Adauri Brezolin for English revision of the manuscript.

\section{Disclosure Statement}

The authors have no conflicts of interest to declare.

\section{References}

1 Thorens B, Charron MJ, Lodish HF: Molecular physiology of glucose transporter. Diabetes Care 1990;13:209-218.

2 Corrêa-Giannella ML, Machado UF: SLC2A4 gene: a promising target for pharmacogenomics of insulin resistance. Pharmacogenomics 2013;14:847-850.

3 Im SS, Kwon SK, Kim TH, Kim HI, Ahn YH: Regulation of glucose transporter type 4 isoform gene expression in muscle and adipocytes. IUBMB Life 2007;59:134-145.

- 4 Liu ML, Olson NP, Edgington WS, Moye-Rowley WS, Pessin JE: Myocyte enhancer factor 2 (MEF2) binding site is essential for C2C12 myotube-specific expression of the rat GLUT4/muscle-adipose facilitative glucose transporter gene. J Biol Chem 1994;269:28514-28521.

- 5 Santalucia T, Moreno H, Palacín M, Yacoub MH, Brand NJ, Zorzano A: A novel functional co-operation between MyoD, MEF2 and TRalpha1 is sufficient for the induction of GLUT4 gene transcription. J Mol Biol 2001;314:195-204.

- 6 Furuya DT, Neri EA, Poletto AC, Anhê GF, Freitas HS, Campello RS, Rebouças NA, Machado UF: Identification of nuclear factor-кB sites in the Slc2a4 gene promoter. Mol Cell Endocrinol 2013;370:87-95.

- 7 Alves-Wagner ABT, Freitas HS, Souza PB, Seraphim PM, Mori RCT, Machado UF: $\beta$-adrenergic activity preserves GLUT4 protein in glycolytic fibers in fasting. Muscle Nerve 2009;40:847-854.

- 8 Alves-Wagner AB, Sabino-Silva R, Campello RS, Mori RC, Machado UF: Decreased diabetes-induced glycemic impairment in WKY and SHR involves enhanced skeletal muscle Slc2a4/GLUT4 expression. Diabetol Metab Syndr 2014;6:97.

9 Cabassi A, Vinci S, Cantoni AM, Quartieri F, Moschini L, Cavazzini S, Cavatorta A, Borghetti A: Sympathetic activation in adipose tissue and skeletal muscle of hypertensive rats. Hypertension 2002;39:656-661.

10 Altarejos JY, Montminy M: CREB and the CRTC co-activators: sensors for hormonal and metabolic signals. Nat Rev Mol Cell Biol 2011;12:141-151. 


\section{Cellular Physiology Cell Physiol Biochem 2019;52:580-594 \begin{tabular}{ll|l} 
and Biochemistry & $\begin{array}{l}\text { DOl: 10.33594/000000041 } \\
\text { Published online: 22 March 2019 }\end{array}$ & $\begin{array}{l}\text { O 2019 The Author(s). Published by } \\
\text { Cell Physiol Biochem Press GmbH\&Co. KG }\end{array}$ \\
\cline { 2 - 3 }
\end{tabular}}

Alves-Wagner et al.: CRE Site in the ScI2a4 Promoter Gene

11 Brivanlou AH, Darnell Jr. JE: Signal transduction and the control of gene expression. Science 2002;295:813818.

- 12 Thomson DM, Herway ST, Fillmore N, Kim H, Brown JD, Barrow JR, Winder WW: AMP-activated protein kinase phosphorylates transcription factors of the CREB family. J Appl Physiol (1985) 2008;104:429-438.

- 13 Sands WA, Palmer TM: Regulating gene transcription in response to cyclic AMP elevation. Cell Signal 2008;30:460-466.

- 14 Berdeaux R, Goebel N, Banaszynski L, Takemori H, Wandless T, Shelton GD, Montminy M: SIK1 is a class II HDAC kinase that promotes survival of skeletal myocytes. Nature 2007;3:597-603.

- 15 Yonamine CY, Pinheiro-Machado E, Michalani ML, Freitas HS, Okamoto MM, Corrêa-Giannella ML, Machado UF: Resveratrol improves glycemic control in insulin-treated diabetic rats: participation of the hepatic territory. Nutr Metab (Lond) 2016;13:44.

16 Moraes PA, Yonamine CY, Pinto Junior DC, Esteves JV, Machado UF, Mori RC: Insulin acutely triggers transcription of Slc2a4 gene: participation of the AT-rich, E-box and NFKB-binding sites. Life Sci 2014;114:36-44.

17 Alves-Wagner AB, Mori RC, Sabino-Silva R, Fatima LA, da Silva Alves A, Britto LR, D’Agord Schaan B, Machado UF: Beta-adrenergic blockade increases GLUT4 and improves glycemic control in insulin-treated diabetic Wistar rats. Auton Neurosci 2015;193:108-116.

18 Montminy MR, Sevarino KA, Wagner JA, Mandel G, Goodman RH: Identification of a cyclic-AMP-responsive element within the rat somatostatin gene. Proc Natl Acad Sci USA 1986;83:6682-6686.

- 19 Zhang X, Odom DT, Seung-Hoi K, Conkright MD, Canettieri G, Best J, Chen H, Jenner R, Herbolsheimer E, Jacobsen E, Kadam S, Ecker JR, Emerson B, Hogenesch JB, Unterman T, Young RA, Montminy M: Genomewide analysis of cAMP-response element binding protein occupancy, phosphorylation, and target gene activation in human tissues. Proc Natl Acad Sci USA 2005;102:4459-4464.

- 20 Qi L, Saberi M, Zmuda E, Wang Y, Altarejos J, Zhang X, Dentin R, Hedrick S, Bandyopadhyay G, Hai T, Olefsky J, Montminy M: Adipocyte CREB promotes insulin resistance in obesity. Cell Metab 2009;9:277-286.

21 Favre D, Gouill EL, Fahmi D, Verdumo C, Chinetti-Gbaguidi G, Staels B, Caiazzo R, Pattou F, Lê K-A, Tappy L, Regazzi R, Giusti V, Vollenweider P, Waeber G, Abderrahmani A: Impaired expression of the inducible cAMP early repressor accounts for sustained adipose CREB activity in obesity. Diabetes 2011;60:3169-3174.

22 Lin B, Morris DW, Chou JY: The role of HNF1alpha, HNF3gamma, and cyclic AMP in glucose-6-phosphatase gene activation. Biochemistry 1997;36:14096-14106.

23 Schmoll D, Wasner C, Hinds CJ, Allan BB, Walther R, Burchell A: Identification of a cAMP response element within the glucose-6-phosphatase hydrolytic subunit gene promoter which is involved in the transcriptional regulation by cAMP and glucocorticoids in H4IIE hepatoma cells. Biochem J 1999;338:457463.

24 Marín-Juez R, Diaz M, Morata J, Planas JV: Mechanisms regulating GLUT4 transcription in skeletal muscle cells are highly conserved across vertebrates. PLoS One 2013;8:e80628.

25 Lima GA, Anhê GF, Giannocco G, Nunes MT, Correa-Giannella ML, Machado UF: Contractile activity per se induces transcriptional activation of SLC2A4 gene in soleus muscle: involvement of MEF2D, HIF-1a, and TRalpha transcriptional factors. Am J Physiol Endocrinol Metab 2009;296:E132-138.

26 Dalen KT, Ulven SM, Bamberg K, Gustafsson J, Nebb HI: Expression of the insulin-responsive glucose transporter GLUT4 in adipocytes is dependent on liver X receptor $\alpha$. J Biol Chem 2003;278:48283-48291.

27 Griesel BA, Weems J, Russel RA, Abel ED, Humphries K, Olson AL: Acute inhibition of fatty acid import inhibits GLUT4 transcription in adipose tissue, but not skeletal or cardiac muscle tissue, partly through liver X receptor (LXR) signaling. Diabetes 2010;59:800-807.

28 Wright JJ, Kim J, Buchanan J, Boudina S, Sena S, Bakirtzi K, Ilkun O, Theobald HÁ, Cooksey RC, Kandror KV, Abel ED: Mechanisms for increased myocardial fatty acid utilization following short-term high-fat feeding. Cardiovasc Res 2009;82:351-360.

29 Atkinson BJ, Griesel BA, King CD, Josey MA, Olson AL: Moderate GLUT4 overexpression improves insulin sensitivity and fasting triglyceridemia in high-fat diet-fed transgenic mice. Diabetes 2013;62:2249-2258.

30 Flores-Riveros JR, Kaestner KH, Thompson KS, Lane MD: Cyclic AMP-induced transcriptional repression of the insulin-responsive glucose transporter (GLUT4) gene: identification of a promoter region required for down-regulation of transcription. Biochem Biophys Res Commun 1993;194:1148-1154. 


\section{Cellular Physiology Cell Physiol Biochem 2019;52:580-594

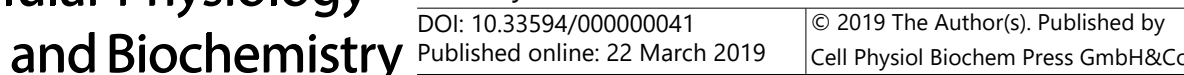 \\ Alves-Wagner et al.: CRE Site in the Scl2a4 Promoter Gene}

31 Viñals F, Ferré J, Fandos C, Santalucia T, Testar X, Palacín M, Zorzano A: Cyclic adenosine 3'5'-monophosphate regulates GLUT4 and GLUT1 glucose transporter expression and stimulates transcriptional activity of the GLUT1 promoter in muscle cells. Endocrinology 1997;138:2521-2529.

32 Cooke DW, Lane MD: Transcription factor NF1 mediates repression of the GLUT4 promoter by cyclic-AMP. Biochem Ciophys Res Commun 1999;260:600-604.

33 Okumura N, Hashida-Okumura A, Kita K, Matsubae M, Matsubara T, Takao T, Nagai K: Proteomic analysis of slow- and fast-twitch skeletal muscles. Proteomics 2005;5:2896-2906.

34 Rakus D, Gizak A, Deshmukh A, Wisniewski JR: Absolute quantitative profiling of the key metabolic pathways in slow and fast skeletal muscle. J Proteome Res 2015;14:1400:11.

35 Zanquetta MM, Nascimento ME, Mori RC, D’Agord Schaan B, Young ME, Machado UF: Participation of betaadrenergic activity in modulation of GLUT4 expression during fasting and refeeding in rats. Metabolism 2006;55:1538-1545.

36 Rubinstein NA, Lyons GE, Kelly AM: Hormonal control of myosin heavy chain genes during development of skeletal muscles. Ciba Found Symp 1988;138:35-51.

37 Festoff BW, Oliver KL, Reddy NB: In vitro studies of skeletal muscle membranes. Adenylate cyclase of fast and slow twitch muscle and the effects of denervation. J Membr Biol 1997;32:331-343.

38 Watson-Wright MW, Wilkinson M: The muscle slice - a new preparation for the characterization of betaadrenergic binding in fast- and slow-twitch skeletal muscle. Muscle Nerve 1986;9:416-422.

39 Martin WH $3^{\text {rd }}$, Murphree SS, Saffitz JE: Beta-adrenergic receptor distribution among muscle fiber types and resistance arterioles of white, red, and intermediated skeletal muscle. Circ Res 1989;64:1096-1105.

- 40 Kim YS, Sainz RD, Molenaar P, Summers RJ: Characterization of beta 1- and 2-adrenoceptors in rat skeletal muscles. Biochem Parmacol 1991;42:1783-1789.

- 41 Collins S, Surwit RS: The beta-adrenergic receptors and the control of adipose tissue metabolism and thermogenesis. Recent Prog Horm Res 2001;56:309-328.

42 Heinonen I, Wendelin-Saarenhovi M, Kaskinoro K, Knuuti J, Scheinin M, Kalliokoski KK: Inhibition of $\alpha$-adrenergic tone disturbs the distribution of blood flow in the exercising human limb. Am J Physiol Heart Circ Physiol 2013;305:H163-H172. 